\title{
Anticancer effect of XAV939 is observed by inhibiting lactose dehydrogenase $A$ in a 3-dimensional culture of colorectal cancer cells
}

\author{
DAHEE KIM ${ }^{1,2^{*}}$, BYUMSEOK KOH $^{1 *}$, KWANG ROK KIM ${ }^{1}$, KI YOUNG KIM ${ }^{1}$, \\ WON HOON JUNG ${ }^{1}$, HI YOUN KIM ${ }^{1}$, SUNGSUB KIM ${ }^{2}$ and SANG DAL RHEE ${ }^{1,2}$ \\ ${ }^{1}$ Therapeutics and Biotechnology Division, Korea Research Institute of Chemical Technology, Yuseong-gu, Daejeon 34114; \\ ${ }^{2}$ Graduate School of New Drug Discovery and Development, Chungnam National University, \\ Yuseong-gu, Daejeon 34134, Republic of Korea
}

Received April 8, 2019; Accepted August 5, 2019

DOI: $10.3892 / \mathrm{ol} .2019 .10813$

\begin{abstract}
XAV939, a tankyrase inhibitor, exerts an anticancer effect in 3-dimensional (3D) cultured SW480 cells, however this is not exhibited in 2-dimensional (2D) cultured SW480 cells. In the current study, XAV939 induced a 3.7-fold increase in cellular apoptosis in 3D culture but not in the 2D culture. However, no significant changes were indicated in cell cycle distribution in the $2 \mathrm{D}$ or $3 \mathrm{D}$ culture. Based on the observation that protein expression, which was associated with the glycolytic pathway, was increased in the 3D culture, the effect of XAV939 on the patterns of glycolytic protein expression was assessed. XAV939 was revealed to decrease lactose dehydrogenase A (LDHA) expression in 3D cultured SW480 cells, but only exerted a small effect in the 2D culture. The coadministration of XAV939 with the LDHA inhibitor FX11 decreased proliferation in 3D cultured SW480 cells compared with the single administration of FX11, while there was no additive effect in the 2D culture. The lactate assay also indicated that XAV939 decreased lactate secretion in the 3D cell culture but not in the 2D culture. These results suggest that XAV939 exerts an anticancer effect through inhibition of LDHA in the 3D culture.
\end{abstract}

\section{Introduction}

There has been growing interest in targeting the Wnt signaling pathway to treat colorectal cancer in the last two decades (1-3).

Correspondence to: Dr Sang Dal Rhee, Therapeutics and Biotechnology Division, Korea Research Institute of Chemical Technology, 141 Gajeong-Ro, Yuseong-gu, Daejeon 34114, Republic of Korea

E-mail: sdrhee@krict.re.kr

*Contributed equally

Key words: 3D culture, XAV939, lactose dehydrogenase A, FX11, synergistic effect
Several anticancer agents that control the Wnt signaling pathway have been reported, including small molecules, antibodies and peptides (4-6); however, there is no Wnt inhibitor approved by the FDA. Chen and colleagues found that tankyrase inhibitors have an antagonistic effect on the Wnt signaling pathway and inhibit colon cancer cell proliferation (7). Tankyrase mediates the poly-ADP-ribosylation of AXIN1 and AXIN2, which are important components of the beta-catenin destruction complex and induce subsequent results in freeing beta-catenin (8-10). Later studies reported that XAV939 inhibits both tankyrase-1 and tankyrase-2, thereby retaining AXIN protein expression (5). In addition, XAV939 inhibits the Wnt signaling pathway in adenomatous polyposis coli (APC)-mutated colon cancer cell lines, COLO-320DM and SW403 (11).

However, our recent study showed that XAV939 is effective in treating SW480 grafted in vivo mouse models and 3-dimensional (3D) cultured models but not monolayer cultures $(12,13)$. Quantitative proteomic analyses of 2-dimensional (2D) and 3D SW480 cultured cells after treatment with XAV939 revealed that the expression levels of glycolytic pathway-related proteins were upregulated in 3D culture compared to 2D culture, while proteins involved in oxidative phosphorylation were downregulated (12). This is one of the hallmarks of metabolic reprogramming, specifically anaerobic glycolysis (Warburg metabolism) (14-16). The study also showed that the expression level of lactose dehydrogenase A (LDHA) is significantly increased in 3D culture compared to 2D culture (12). Because LDHA is also closely related to glycolytic processes (17-19), it would be intriguing to find a relationship between the different anticancer effects of XAV939 on 2D/3D cell culture and changes in glycolytic metabolism. Here, we report systematic comparison studies of 2D and 3D SW480 cell culture focusing on glycolytic pathways in each culture system to determine the mechanism behind this different response against XAV939.

\section{Materials and methods}

Reagents. The SW480 colon cancer cell line was obtained from the American Type Culture Collection (ATCC, VA, USA). RPMI-1640 medium powder, penicillin-streptomycin 
and protease inhibitor cocktail were purchased from Thermo Fisher Scientific, Inc. (MA, USA). XAV939 (98\%, HPLC) as purchased from Sigma-Aldrich (MO, USA). FX11 was purchased from Merck Millipore (MA, USA).

$2 D$ and $3 D$ culture. SW480 cells were cultured in RPMI-1640 medium supplemented with $10 \%$ FBS and $100 \mathrm{U} / \mathrm{ml}$ penicillin and $100 \mu \mathrm{g} / \mathrm{ml}$ streptomycin at $37^{\circ} \mathrm{C}$ in a $5 \% \mathrm{CO}_{2}$ incubator. Cells from passage numbers $<15$ were used throughout the experiment. For. 3D culture, a Cytoselect 96-well transformation assay kit (Cell Biolabs, Inc., CA, USA) was used. After agar layer solidification, RPMI-1640 medium was placed on top of the 3D culture and cultured for 6-8 days. XAV939 $(20 \mu \mathrm{M})$ in DMSO was added to cells after $24 \mathrm{~h}$ of stabilization. Then, RPMI-1640 medium containing XAV939 was replaced every $48 \mathrm{~h}$.

Western blot analyses. Cells were lysed in ice-cold whole-cell extract buffer ( $\mathrm{pH}$ 7.4) containing protease inhibitors. The protein concentrations were determined by a BCA protein assay kit (Pierce, IL, USA). Equal amounts of protein were subjected to electrophoresis using $10 \%$ sodium dodecyl sulfate-polyacrylamide gels (Bio-Rad Laboratories, Inc., CA, USA). After transfer to polyvinylidene fluoride membranes (Bio-Rad Laboratories, Inc.), proteins were sequentially hybridized with a primary antibody (AXIN2, cyclin D1,CDK2, CDK4, p21, p27, PGK1, GAPDH, LDHA and c-Myc, Cell Signaling Technology, MA, USA) followed by an HRP-conjugated second antibody (Thermo Fisher Scientific, Inc.). Protein bands were visualized by ImageQuant LAS500 (GE Healthcare, IL, USA). $\beta$-Actin (Sigma-Aldrich) was used as the protein loading control.

Effect of XAV939 on 2D/3D SW480 cell proliferation. For $2 \mathrm{D}$ culture, $2 \times 10^{5}$ cells/well were seeded into a 6 -well plate, and XAV939 was added after $24 \mathrm{~h}$. Two hundred microliters of CCK-8 (Dojindo Laboratories, Japan) was added to the cell culture after $0,1,2$ and 3 days of XAV939 treatment. Absorbance at $450 \mathrm{~nm}$ from formazan byproducts was measured using a SpectraMax MiniMax 300 imaging cytometer (Molecular Devices, CA, USA). For 3D cell culture, mixture of agar/SW480 cells were seeded into 96-well plates, and XAV939 was added after $24 \mathrm{~h}$. RPMI-1640 medium was replaced every $48 \mathrm{~h}$ and incubated for 2, 4 and 6 days. After incubation, the 3D agar cell layer was solubilized with the addition of $50 \mu \mathrm{l}$ of agar solubilization solution, and cell proliferation was quantified using CyQuant dye and fluorescence at $520 \mathrm{~nm}$. To measure apoptosis, a Muse Annexin V and Dead Cell Assay Kit was used and analyzed with a Muse cell analyzer (Merck Millipore, MA, USA).

XAV939 on the 2D/3D SW480 cell cycle. To determine the effect of XAV939 on the cell cycle in 2D and 3D culture, assays were performed using the Muse cell cycle kit. In brief, cells were fixed with $70 \%$ ethanol and stored at $-20^{\circ} \mathrm{C}$. Fixed cells were centrifuged, and cell cycle reagent was added and incubated for $30 \mathrm{~min}$ at room temperature and quantified using a Muse cell analyzer. To determine the effect of XAV939 on glycolytic protein expression, 2D/3D cells after XAV939 treatment were solubilized with cell lysis buffer (8M urea, $50 \mathrm{mM}$ Tris- $\mathrm{HCl}$ ( $\mathrm{pH} 8.0), 75 \mathrm{mM} \mathrm{NaCl}$, and protease inhibitor). Solubilized proteins were loaded onto precast SDS-PAGE gels and detected with ECL reagent on an ImageQuant ${ }^{\mathrm{TM}}$ LAS 500 imager (GE Healthcare, IL, USA).

FX11 and XAV939 on 2D/3D SW480 cell growth. 2D/3D cell cultures were prepared as described above. Various concentrations $(1,5,10,20,50$, and $100 \mu \mathrm{M})$ of FX11 or FX11 together with $20 \mu \mathrm{M}$ XAV939 were administered to the 2D/3D cell culture, and cell culture media containing FX11 or FX11/XAV939 was replaced every $48 \mathrm{~h}$. Growth inhibition of the 2D/3D culture was measured with CCK-8.

Lactate assay. Lactate concentration was measured using an EZ-Lactate Assay Kit (DogenBio, Korea). In brief, 8x10 2D/3D cells were treated with $20 \mu \mathrm{M}$ XAV939, and LDH was removed from the supernatant using a deproteinizing sample preparation kit (BioVision, CA, USA). LDH-removed supernatant was reacted with water-soluble tetrazolium salts (WST) for $30 \mathrm{~min}$ at room temperature. Absorbance at $450 \mathrm{~nm}$ was measured using SpectraMax.

Statistical analyses. Statistical analysis was performed using GraphPad Prism (version 6, GraphPad Software, Inc., CA, USA). Each experiment was performed in triplicate, and values are expressed as the mean \pm standard deviation (SD). Statistical significance were examined by Student's t-test, one-way ANOVA, and two-way ANOVA. After completing ANOVAs, Tukey's post-hoc test was conducted for pairwise comparisons. Statistical significance is denoted as $*$ for $\mathrm{P}<0.05$, ** for $\mathrm{P}<0.01$, and $* * *$ for $\mathrm{P}<0.001$.

\section{Results}

Effect of XAV939 on 2D/3D SW480 cell proliferation and apoptosis. To confirm the different anticancer abilities of XAV939 on 2D/3D SW480 cell culture, the time-dependent effect of XAV939 on 2D/3D cell growth and apoptosis was monitored. XAV939 did not induce an antiproliferative effect on $2 \mathrm{D}$ culture, as cell proliferation was $96.6 \pm 1.1,93.0 \pm 3.3$ and $102.9 \pm 0.8 \%$ at 1,2 and 3 days after the addition of $20 \mu \mathrm{M}$ XAV939 compared to the control (Fig. 1A). In contrast, $20 \mu \mathrm{M}$ XAV939 induced a 15.7 $\pm 1.4(\mathrm{P}<0.05), 26.6 \pm 4.3(\mathrm{P}<0.001)$ and $32.2 \pm 3.9 \%(\mathrm{P}<0.001)$ decrease in $3 \mathrm{D}$ cell proliferation compared to the control (Fig. 1B). The $\%$ of apoptotic cells in 3D culture showed that the apoptotic rate of SW480 cells increased by $3.7 \pm 0.4(\mathrm{P}<0.01)$-fold upon the addition of $20 \mu \mathrm{M}$ XAV939 (Fig. 1C), while the apoptotic rate in 2D culture was increased by $0.8 \pm 0.2(\mathrm{P}<0.01)$-fold with the same concentration of XAV939 (Fig. 1D). Western blot analysis showed an increase in AXIN2 expression in 2D/3D culture while demonstrating a decrease in $\beta$-catenin expression in $2 \mathrm{D} / 3 \mathrm{D}$ culture upon $20 \mu \mathrm{M}$ XAV939 treatment, indicating inhibition of the Wnt signaling pathway (Fig. 1E).

Effect of XAV939 on cell cycle protein expression in $2 D / 3 D$ SW480 cell culture. To determine whether XAV939 exerts an effect on the cell cycle of 2D/3D cultured SW480 cells, the expression levels of proteins related to the cell cycle were monitored upon the addition of XAV939. No significant changes in the cell cycle of $2 \mathrm{D}$ or $3 \mathrm{D}$ cultured cells were observed after XAV939 treatment (Fig. 2A). XAV939 does 
A
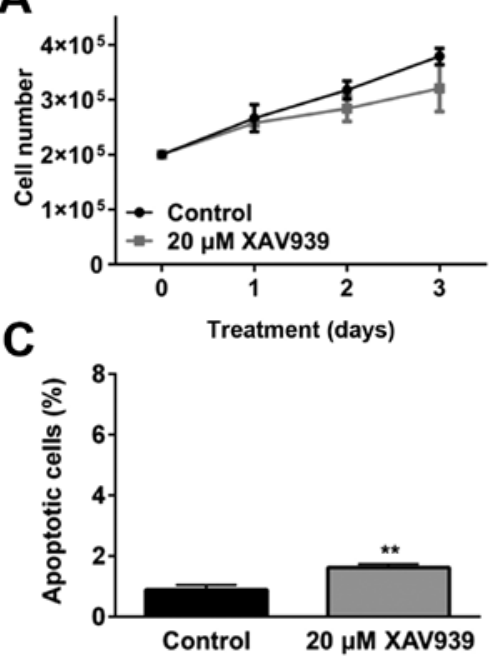

$\mathbf{E}$

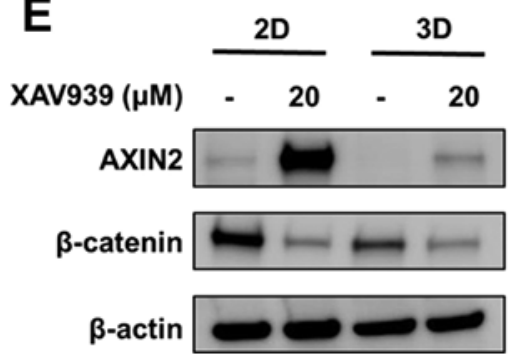

B
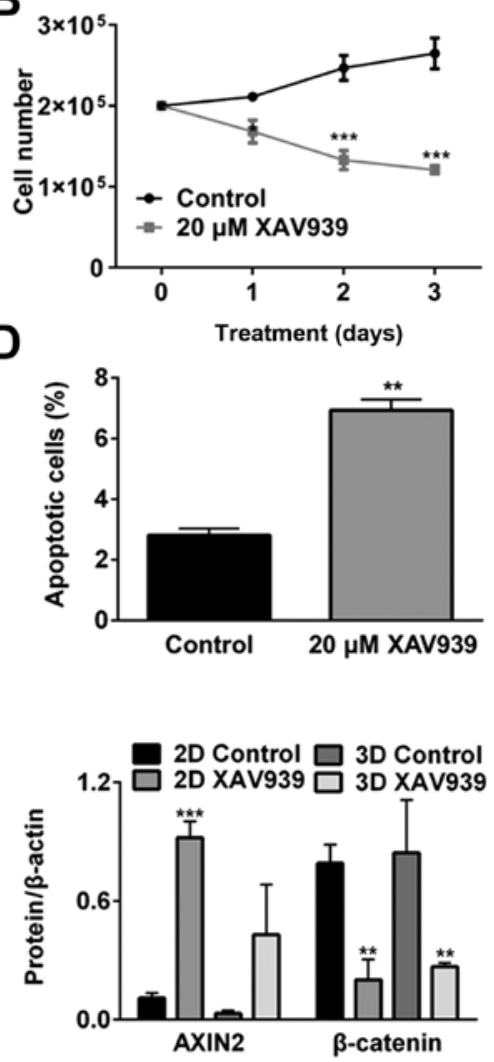

Figure 1. Effects of XAV939 on the growth of SW480 cells. Time-dependent effect of XAV939 on (A) 2D and (B) 3D cultured SW480 cell proliferation. Effect of XAV939 on the apoptosis of (C) 2D and (D) 3D SW480 cells. (E) Protein expression of AXIN2 and $\beta$-catenin were examined using western blot analysis in 2D and 3D SW480 cells. The quantification of band intensities was performed using ImageJ. The amount of proteins (AXIN2 and $\beta$-catenin) on the $\mathrm{x}$-axis was calculated relative to the $\beta$-actin protein level. The results are expressed as the mean \pm standard deviation from triplicate experiments. Data were statistically analyzed using a two-way ANOVA and a t-test. ${ }^{* *} \mathrm{P}<0.01,{ }^{* * * *} \mathrm{P}<0.001$ vs. the control group. 2D, 2-dimensional; 3D, 3-dimensional.

A

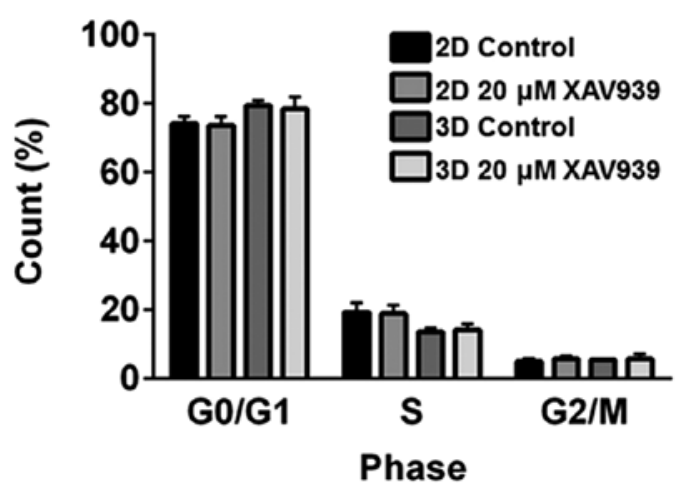

B
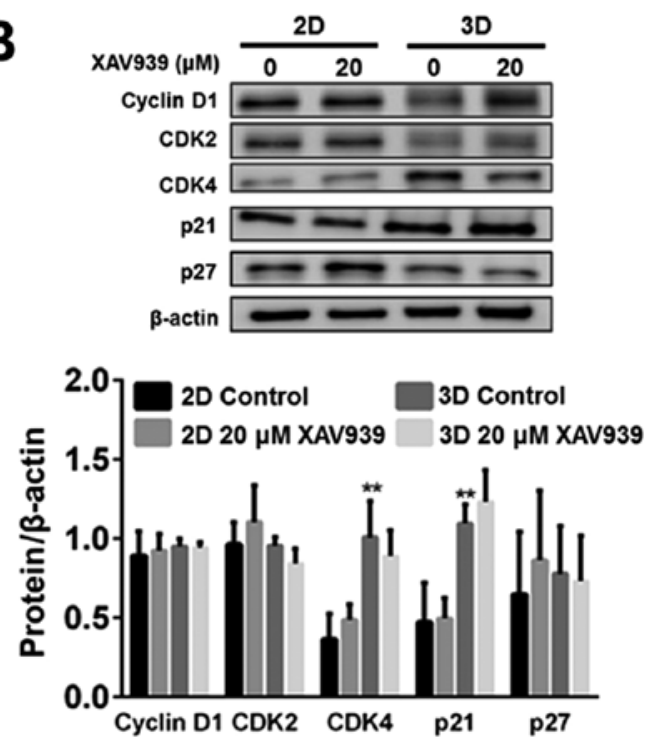

Figure 2. Effects of XAV939 on the cell cycle. (A) Cell cycle assay in the 2D and 3D SW480 cell culture. (B) The expression of proteins associated with the cell cycle were examined using western blot analysis in 2D and 3D cultured SW480 cells after XAV939 treatment. The quantification of band intensities was performed using ImageJ. The amount of proteins on the $\mathrm{x}$-axis was calculated relative to the $\beta$-actin protein level. The results are expressed as the mean \pm SD from triplicate experiments. Data were statistically analyzed by two-way ANOVA. ${ }^{* *} \mathrm{P}<0.01$ vs. $2 \mathrm{D}$ the control group. $2 \mathrm{D}, 2$-dimensional; 3D, 3-dimensional.

not induce significant changes in cell cycle-related protein levels, as the expression of cyclin D1, CDK2, p21 and p27 does not differ upon the addition of XAV939 in either 2D or 3D culture (Fig. 2B). Only a $1.8 \pm 0.2$ - and a $2.2 \pm 0.8$-fold increase in CDK4 and p21 expression levels in the 3D cultured control was observed compared to the $2 \mathrm{D}$ cultured control. 
A

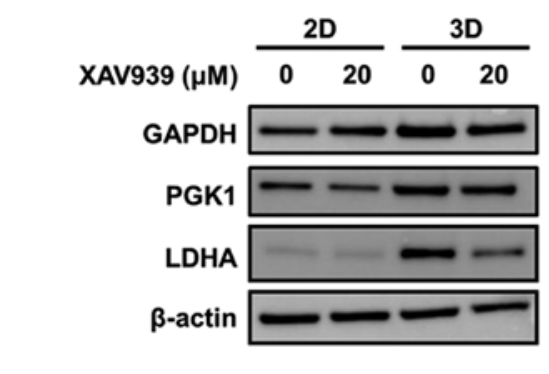

C

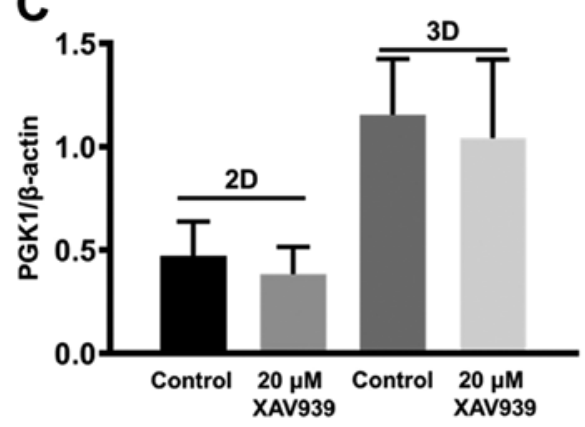

B

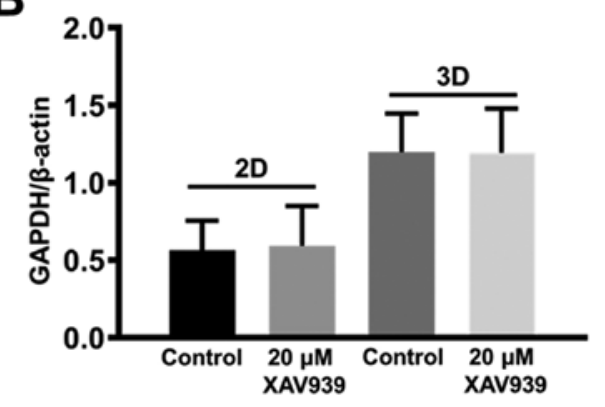

D

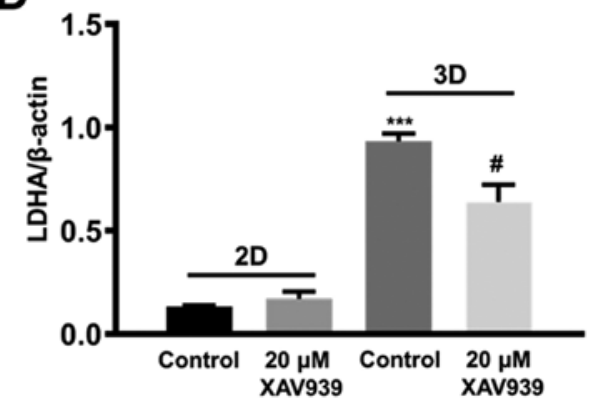

Figure 3. Effects of XAV939 on glycolytic protein expression. (A) The protein expression of GAPDH, PGK1 and LDHA were examined using western blot analysis in 2D and 3D cultured SW480 cells. The quantification of band intensities was performed using ImageJ. (B) GAPDH protein expression. (C) PGK1 protein expression. (D) LDHA protein expression. The amount of proteins on the $\mathrm{x}$-axis was calculated relative to the $\beta$-actin protein level. The results are expressed as the mean \pm standard deviation from triplicate experiments. Data were statistically analyzed using a one-way ANOVA. ${ }^{* * *} \mathrm{P}<0.001$ vs. the $2 \mathrm{D}$ control group. ${ }^{~} \mathrm{P}<0.01$ vs. the 3D control group. LDHA, lactose dehydrogenase A; 2D, 2-dimensional; 3D, 3-dimensional.

Effect of XAV939 on glycolytic enzyme expression in $2 D / 3 D$ SW480 cell culture. When the expression levels of glycolysis-associated proteins were examined, the levels of GAPDH, PGK1 and LDHA were increased in 3D culture compared to 2D culture (Fig. 3A-D). In 2D culture, no significant changes in glycolysis-related protein expression level were observed upon XAV939 treatment; however, in 3D culture, the expression level of LDHA was decreased by $34.7 \pm 7.1 \%(\mathrm{P}<0.05)$ compared to the control. Although the expression of $\mathrm{c}-\mathrm{Myc}$ was decreased in both 2D and 3D culture, the expression level decreased by $8.3 \pm 0.5 \%$ in $2 \mathrm{D}$ culture $(\mathrm{P}>0.05)$, while $3 \mathrm{D}$ cultured cells showed a $28.9 \pm 2.1 \%(\mathrm{P}<0.05)$ decrease upon the addition of XAV939 (Fig. 4A and B).

Effects of LDHA inhibition on the proliferation of SW480 cells. Various concentrations $(1-100 \mu \mathrm{M})$ of the LDHA inhibitor FX11 were applied to examine the effect on LDHA inhibition in 2D/3D cultured SW480 cells. An FX11 concentration-dependent decrease in proliferation was observed in 2D/3D culture at FX11 concentrations greater than $20 \mu \mathrm{M}$ (Fig. 5A). The $\mathrm{IC}_{50}$ of FX11 in the $2 \mathrm{D}$ and $3 \mathrm{D}$ cultures was 67.5 and $65.3 \mu \mathrm{M}$, respectively, and the effect of FX11 on 2D/3D cultured cell growth only differed by $4.2 \pm 0.4 \%$. Based on the effect of FX11 on 2D/3D cultured cell growth, various concentrations of FX11 were coadministered with $20 \mu \mathrm{M}$ XAV939 to determine the effect of XAV939 on LDHA inhibition. The coadministration of $20 \mu \mathrm{M}$ XAV939 with FX11 did not induce changes in 2D cultured cell proliferation compared to FX11 single administration (Fig. 5B). However, XAV939 induced additive inhibition in 3D cultured cell proliferation when cotreated with FX11. For an effective comparison, $20 \mu \mathrm{M}$ XAV939 and FX11 were added to the 2D/3D culture,
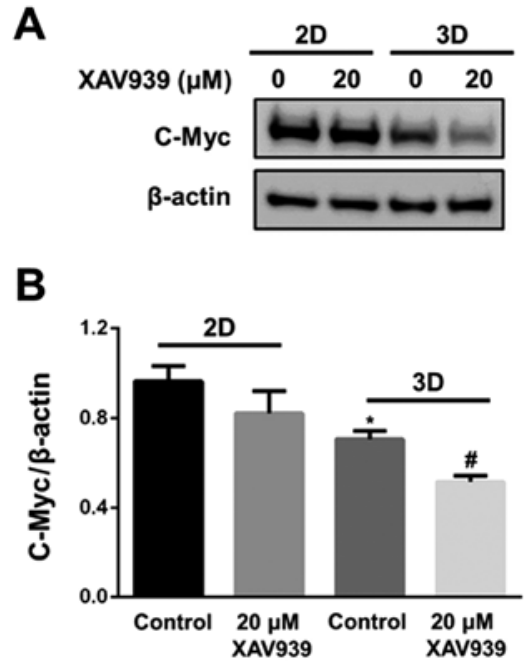

Figure 4. Effects of XAV939 on c-Myc protein expression. (A) The protein expression of c-Myc were examined using western blot analysis in 2D and 3D cultured SW480 cells. (B) The quantification of band intensities was performed using ImageJ. The amount of proteins on the $\mathrm{x}$-axis was calculated relative to the $\beta$-actin protein level. The results are expressed as the mean \pm standard deviation from triplicate experiments. Data were statistically analyzed using a one-way ANOVA. ${ }^{*} \mathrm{P}<0.05$ vs. the $2 \mathrm{D}$ control group. ${ }^{\#} \mathrm{P}<0.05$ vs. the $2 \mathrm{D}$ control group. $2 \mathrm{D}, 2$-dimensional; 3D, 3-dimensional.

and cell proliferation was monitored. In the $2 \mathrm{D}$ culture, $20 \mu \mathrm{M}$ FX11 induced a $14.7 \pm 2.3 \%$ decrease, while the coadministration of $20 \mu \mathrm{M}$ XAV939/FX11 induced a $14.2 \%$ decrease in proliferation (Fig. 5C). In contrast, $20 \mu \mathrm{M}$ FX11 induced a $23.8 \pm 2.9 \%$ decrease, while the coadministration of $20 \mu \mathrm{M}$ XAV939/FX11 induced a $51.5 \pm 4.0 \%(\mathrm{P}<0.001)$ decrease in 3D culture proliferation (Fig. 5C). We also confirmed that $5 \mu \mathrm{M}$ of 

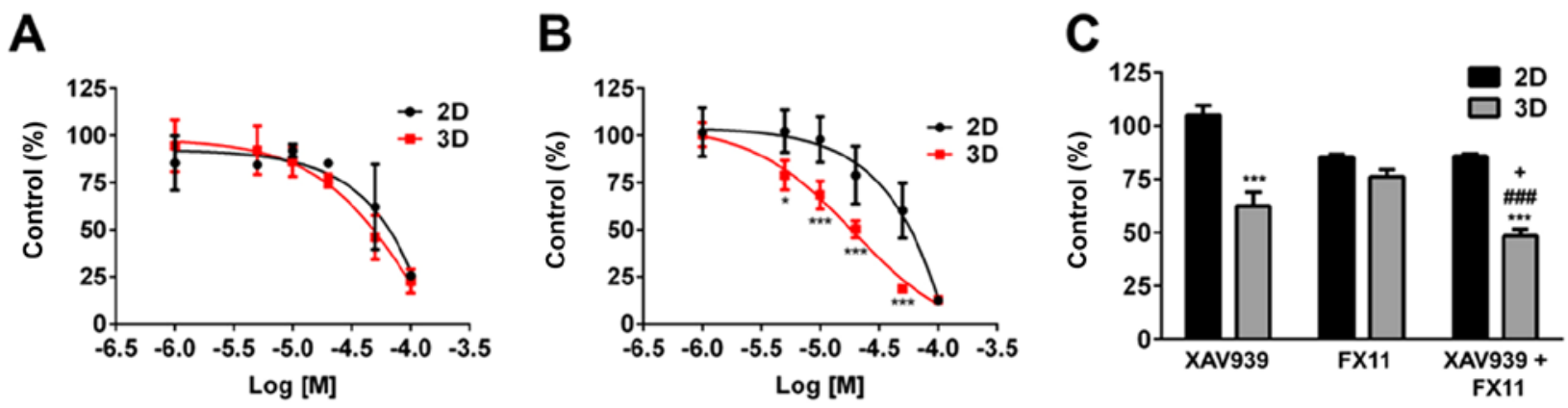

Figure 5. Effects of LDHA inhibition on the growth of SW480 cells. (A) Effect of indicated concentrations of FX11 on 2D and 3D cultured SW480 viability. (B) Effect of the combination of $20 \mu \mathrm{M}$ XAV939 and the indicated concentrations of FX11 on cell growth. (C) Effect of combined treatment of $20 \mu \mathrm{M}$ FX11 and $20 \mu \mathrm{M}$ XAV939 on viability of 2D and 3D cultured SW480. The results are expressed as the mean \pm standard deviation from triplicate experiments. Data were statistically analyzed using a two-way ANOVA. ${ }^{*} \mathrm{P}<0.05$ and ${ }^{* * * *} \mathrm{P}<0.001$ vs. the $2 \mathrm{D}$ group. ${ }^{\# \#} \mathrm{P}<0.001$ vs. the $3 \mathrm{D}$ FX11 group. ${ }^{+} \mathrm{P}<0.05$ vs. the $3 \mathrm{D} \mathrm{XAV} 939$ group. LDHA, lactose dehydrogenase A; 2D, 2-dimensional; 3D, 3-dimensional.

FX11 induce little or no decrease in LDHA expression level of 2D and 3D SW480 (Fig. S1A). We also conduct experiment of lactation secretion and same concentration of FX11 induces only 2.3 and $8.7 \%$ decrease in lactation secretion in $2 \mathrm{D}$ and 3D SW480 culture respectively (Fig. S1B). Cell viability of 2D and 3D SW480 were not significantly decrease upon addition of 1-20 $\mu \mathrm{M}$ FX11 (Fig. S1C).

Effects of XAV939 on lactate secretion. To examine the relationship between the effect of XAV939 on LDHA inhibition and lactate secretion, a lactate assay was performed. In the $2 \mathrm{D}$ culture system, lactation secretion after $20 \mu \mathrm{M}$ XAV939 administration was $106.3 \pm 10.4 \mathrm{nmol}$, while that of the control was $107.5 \pm 6.1 \mathrm{nmol}$ (Fig. 6). In contrast, lactation secretion after $20 \mu \mathrm{M}$ XAV939 treatment was $130.3 \pm 2.8 \mathrm{nmol}(\mathrm{P}<0.05$ compared to the 3D non-XAV939-treated control), while that of the non-XAV939-treated control was $161.7 \pm 10.4 \mathrm{nmol}$ in 3D culture (Fig. 6).

\section{Discussion}

Previous studies have shown that the tankyrase inhibitor XAV939 is potent in inhibiting 3D but not 2D cultured SW480 cells (12). This study focused on the mechanism behind the different anticancer effects of XAV939 on 2D/3D cultured SW480 cells. We first confirmed the effect of XAV939 on SW480 cell viability in 2D and 3D cultures. We specifically used $20 \mu \mathrm{M}$ XAV939, because our previous study shows that at this concentration, protein expression pattern changed most drastically (12). In 2D culture, $20 \mu \mathrm{M}$ XAV939 induced no significant decrease in cell viability until day 3 . However, the same concentration of XAV939 induced a decrease in 3D cultured cell viability beginning on day 1 (Fig. 1A and B). This result provides evidence that the different effects of XAV939 on SW480 cells are not due to the culturing period difference between 2D and 3D cell culture. As Kim et al showed that the protein expression level of AXIN2 increased and the expression level of $\beta$-catenin decreased in $2 \mathrm{D}$ and $3 \mathrm{D}$ cultured cells upon XAV939 treatment (12), we concluded that XAV939 is effective in inhibiting the Wnt signaling pathway in both 2D and 3D culture. To determine other possible mechanisms involved, we examined the effect of XAV939 on apoptosis and the cell cycle in 2D/3D culture. The apoptotic ratio of

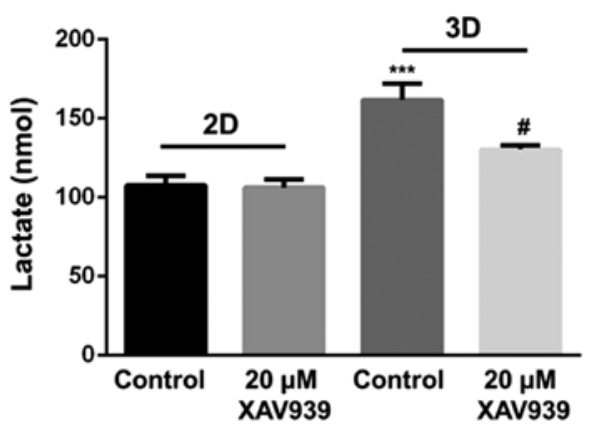

Figure 6. Effects of XAV939 on lactate secretion. Effects of XAV939 on lactate secretion in culture media. ${ }^{* * *} \mathrm{P}<0.001$ vs. the $2 \mathrm{D}$ control group. ${ }^{\#} \mathrm{P}<0.05$ vs. the $3 \mathrm{D}$ control group. The results are expressed as the mean \pm standard deviation from triplicate experiments. Data were statistically analyzed using a two-way ANOVA. 2D, 2-dimensional; 3D, 3-dimensional.

2D cultured cells increased $\sim 1 \%$, while the apoptotic ratio of 3D cultured cells increased by $\sim 5 \%$ upon XAV939 treatment (Fig. 1C and D). XAV939 does not induce significant changes in the $2 \mathrm{D} / 3 \mathrm{D}$ cell cycle, as the data show only a slight increase in the G0/G1 phase and a slight decrease in the S phase in 3D culture (Fig. 2A). A study by Wu et al suggests that XAV939 does not change the cell cycle (4), while other studies show that XAV939 induces apoptosis in cancer cells $(20,21)$. Based on these results, we presumed that XAV939 decreases 3D cultured SW480 cell viability by inducing apoptosis independent of the Wnt signaling pathway.

Our previous proteomic analysis of SW480 cells after XAV939 treatment showed that proteins related to glycolysis are upregulated and proteins related to oxidative phosphorylation are downregulated in $3 \mathrm{D}$ culture compared to $2 \mathrm{D}$ culture, which is genotypically representative of Warburg metabolism $(22,23)$. In addition, $2 \mathrm{D}$ and 3D cell culture showed different expression patterns of LDHA after XAV939 treatment. Because LDHA is one of the key enzymes in Warburg metabolism that is involved in the conversion of pyruvate into lactic acid $(24,25)$, we assumed that XAV939 exerts its effect by manipulating anaerobic glycolysis in 3D culture, thus inducing apoptosis and inhibiting cancer cell growth. In Warburg metabolism, adenosine triphosphate (ATP) is produced through anaerobic glycolysis. Therefore, the abnormal expression of proteins related to glycolysis is 
often observed (26). We examined the expression levels of proteins involved in glycolysis, and the expression levels of GAPDH and PGK1 were increased in 3D culture compared to 2D culture; however, no significant changes in those proteins after XAV939 treatment were observed in either 2D or 3D culture (Fig. 3A-C). In contrast, the expression level of LDHA was significantly decreased in 3D culture but only after XAV939 treatment (Fig. 3D). As studies by other groups also suggest no changes in the LDHA level upon XAV939 treatment in $2 \mathrm{D}$ cell culture, we concluded that this decrease in LDHA expression level is 3D culture-specific $(27,28)$. Next we examine the expression level of c-myc, which is an upstream protein together with LDHA in the glycolysis pathway and Wnt signaling target gene to figure out the relationship with XAV939 (29). The protein expression level of c-myc was also significantly decreased in 3D culture but only after XAV939 treatment (Fig. 4). SW480 cells were treated with the LDHA selective inhibitor FX11 to observe an effect of LDHA inhibition on cell growth (Fig. 5). The viability of both $2 \mathrm{D}$ and $3 \mathrm{D}$ cultured cells decreased with increasing FX11 concentrations; however, we observed an additive effect when cotreated with XAV939 in 3D culture (Fig. 5B and C). Co-administration of XAV939 and FX11 on LDHA expression and lactate secretion suggesting that although FX11 induce small decrease in LDHA expression and lactate secretion, combination with XAV939 shows synergistic effect (Fig. S1). A previous study showed that XAV939 induces a decrease in pyruvate dehydrogenase kinase (PDK) and inhibits the metabolic process of cancer, which heavily relies on anaerobic glycolysis (30). Le et al also suggested that the inhibition of LDHA induces oxidative stress in cancer cells, resulting in apoptosis and cell death (18). We observed a statistically significant decrease in lactate production in 3D culture but only after XAV939 treatment (Fig. 6), which can be correlated with a decrease in LDHA expression level upon XAV939 treatment in 3D culture. However, the detailed mechanism of XAV939 and LDHA downregulation needs to be elucidated. In addition, only MDA-MB-468 cell line was used in the present study and we are currently investigating additional cell lines to verify the findings.

In summary, our results suggest that XAV939 specifically inhibits LDHA and decreases lactate secretion in 3D SW480 cell culture. The inhibition of glycolytic metabolism increases apoptosis and decreases cell viability in $3 \mathrm{D}$ culture upon XAV939 treatment.

\section{Acknowledgements}

The authors would like to thank Dr Young Eun Kim and Dr Dukjin Kang (both from the Korea Research Institute of Standards and Science, Daejeon, Republic of Korea) for aiding in proteomic data acquisition.

\section{Funding}

The present study was financially supported by the R\&D Convergence Program (grant no. CAP1510KRICT), the DRC program (grant no. DRC 15-01-KRICT) of the National Research Council of Science and Technology and the core project (grant no. KK1703-F00) of the Korea Research Institute of Chemical Technology.

\section{Availability of data and materials}

The datasets used during the present study are available from the corresponding author upon reasonable request.

\section{Authors' contributions}

DK, KYK, KRK, SK and SDR conceived and designed the study. DK, BK, WHJ and HYK performed the experiments. DK and BK wrote the manuscript. DK, BK and SDR reviewed and edited the manuscript. All authors read and approved the manuscript and agree to be accountable for all aspects of the research in ensuring that the accuracy or integrity of any part of the report are appropriately investigated and resolved.

\section{Ethics approval and consent to participate}

Not applicable.

\section{Patient consent for publication}

Not applicable.

\section{Competing interest}

The authors declare that they have no competing interests.

\section{References}

1. Li VS, Ng SS, Boersema PJ, Low TY, Karthaus WR, Gerlach JP, Mohammed S, Heck AJ, Maurice MM, Mahmoudi T and Clevers $\mathrm{H}$ : Wnt signaling through inhibition of $\beta$-catenin degradation in an intact Axin1 complex. Cell 149: 1245-1256, 2012.

2. Schatoff EM, Leach BI and Dow LE: Wnt Signaling and Colorectal Cancer. Curr Colorectal Cancer Rep 13: 101-110, 2017.

3. Zhan T, Rindtorff $\mathrm{N}$ and Boutros $\mathrm{M}$ : Wnt signaling in cancer. Oncogene 36: 1461-1473, 2017.

4. Wu X, Luo F, Li J, Zhong X and Liu K: Tankyrase 1 inhibitior XAV939 increases chemosensitivity in colon cancer cell lines via inhibition of the Wnt signaling pathway. Int J Oncol 48: 1333-1340, 2016

5. Huang SM, Mishina YM, Liu S, Cheung A, Stegmeier F, Michaud GA, Charlat O, Wiellette E, Zhang Y, Wiessner S, et al: Tankyrase inhibition stabilizes axin and antagonizes Wnt signalling. Nature 461: 614-620, 2009.

6. Liu J, Pan S, Hsieh MH, Ng N, Sun F, Wang T, Kasibhatla S, Schuller AG, Li AG, Cheng D, et al: Targeting Wnt-driven cancer through the inhibition of Porcupine by LGK974. Proc Natl Acad Sci USA 110: 20224-20229, 2013.

7. Chen B, Dodge ME, Tang W, Lu J, Ma Z, Fan CW, Wei S, Hao W, Kilgore J, Williams NS, et al: Small molecule-mediated disruption of Wnt-dependent signaling in tissue regeneration and cancer. Nat Chem Biol 5: 100-107, 2009.

8. Waaler J, Machon O, Tumova L, Dinh H, Korinek V, Wilson SR, Paulsen JE, Pedersen NM, Eide TJ, Machonova O, et al: A novel tankyrase inhibitor decreases canonical Wnt signaling in colon carcinoma cells and reduces tumor growth in conditional APC mutant mice. Cancer Res 72: 2822-2832, 2012.

9. Lau T, Chan E, Callow M, Waaler J, Boggs J, Blake RA, Magnuson S, Sambrone A, Schutten M, Firestein R, et al: A novel tankyrase small-molecule inhibitor suppresses APC mutation-driven colorectal tumor growth. Cancer Res 73: 3132-3144, 2013.

10. Morrone S, Cheng Z, Moon RT, Cong F and Xu W: Crystal structure of a Tankyrase-Axin complex and its implications for Axin turnover and Tankyrase substrate recruitment. Proc Natl Acad Sci USA 109: 1500-1505, 2012. 
11. Tanaka N, Mashima T, Mizutani A, Sato A, Aoyama A, Gong B, Yoshida H, Muramatsu Y, Nakata K, Matsuura M, et al: APC mutations as a potential biomarker for sensitivity to tankyrase inhibitors in colorectal cancer. Mol Cancer Ther 16: 752-762, 2017.

12. Kim YE, Jeon HJ, Kim D, Lee SY, Kim KY, Hong J, Maeng PJ, Kim KR and Kang D: Quantitative proteomic analysis of 2D and 3D cultured colorectal cancer cells: Profiling of tankyrase inhibitor XAV939-induced proteome. Sci Rep 8: 13255,2018

13. Koh B, Jeon H, Kim D, Kang D and Kim KR: Effect of fibroblast co-culture on the proliferation, viability and drug response of colon cancer cells. Oncol Lett 17: 2409-2417, 2019.

14. Lunt SY and Vander Heiden MG: Aerobic glycolysis: meeting the metabolic requirements of cell proliferation. Annu Rev Cell Dev Biol 27: 441-464, 2011.

15. Kuipers EJ, Grady WM, Lieberman D, Seufferlein T, Sung JJ, Boelens PG, van de Velde CJ and Watanabe T: Colorectal cancer. Nat Rev Dis Primers 1: 15065, 2015.

16. Hanahan D and Weinberg RA: Hallmarks of cancer: The next generation. Cell 144: 646-674, 2011.

17. Ooi AT and Gomperts BN: Molecular Pathways: Targeting cellular energy metabolism in cancer via inhibition of SLC2A1 and LDHA. Clin Cancer Res 21: 2440-2444, 2015.

18. Le A, Cooper CR, Gouw AM, Dinavahi R, Maitra A, Deck LM, Royer RE, Vander Jagt DL, Semenza GL and Dang CV: Inhibition of lactate dehydrogenase A induces oxidative stress and inhibits tumor progression. Proc Natl Acad Sci USA 107: 2037-2042, 2010.

19. Boroughs LK and DeBerardinis RJ: Metabolic pathways promoting cancer cell survival and growth. Nat Cell Biol 17: 351-359, 2015.

20. Guo W, Shen F, Xiao W, Chen J and Pan F: Wnt inhibitor XAV939 suppresses the viability of small cell lung cancer NCI-H446 cells and induces apoptosis. Oncol Lett 14: 6585-6591, 2017.

21. Tian XH, Hou WJ, Fang Y, Fan J, Tong H, Bai SL, Chen Q, Xu H and Li Y: XAV939, a tankyrase 1 inhibitior, promotes cell apoptosis in neuroblastoma cell lines by inhibiting Wnt/ $\beta$-catenin signaling pathway. J Exp Clin Cancer Res 32: 100, 2013.
22. Pavlova NN and Thompson CB: The Emerging Hallmarks of Cancer Metabolism. Cell Metab 23: 27-47, 2016.

23. Dang CV, Le A and Gao P: MYC-induced cancer cell energy metabolism and therapeutic opportunities. Clin Cancer Res 15: 6479-6483, 2009.

24. Flores A, Sandoval-Gonzalez S, Takahashi R, Krall A, Sathe L, Wei L, Radu C, Joly JH, Graham NA, Christofk HR and Lowry WE: Increased lactate dehydrogenase activity is dispensable in squamous carcinoma cells of origin. Nat Commun 10: 91, 2019.

25. He TL, Zhang YJ, Jiang H, Li XH, Zhu H and Zheng KL: The c-Myc-LDHA axis positively regulates aerobic glycolysis and promotes tumor progression in pancreatic cancer. Med Oncol 32: $187,2015$.

26. Pelicano H, Martin DS, Xu RH and Huang P: Glycolysis inhibition for anticancer treatment. Oncogene 25: 4633-4646, 2006.

27. Ono M, Yin P, Navarro A, Moravek MB, Coon V JS, Druschitz SA, Gottardi CJ and Bulun SE: Inhibition of canonical WNT signaling attenuates human leiomyoma cell growth. Fertil Steril 101: 1441-1449, 2014.

28. Esen E, Chen J, Karner CM, Okunade AL, Patterson BW and Long F: WNT-LRP5 signaling induces Warburg effect through mTORC2 activation during osteoblast differentiation. Cell Metab 17: 745-755, 2013.

29. He TL, Zhang YJ, Jiang H, Li XH, Zhu H, Zheng KL: The c-Myc-LDHA axis positively regulates aerobic glycolysis and promotes tumor progression in pancreatic cancer. Med Oncol 32: 187,2015

30. PateKT,StringariC,Sprowl-TanioS,WangK,TeSlaa T,HoverterNP, McQuade MM, Garner C, Digman MA, Teitell MA, et al: Wnt signaling directs a metabolic program of glycolysis and angiogenesis in colon cancer. EMBO J 33: 1454-1473, 2014.

This work is licensed under a Creative Commons

Attribution-NonCommercial-NoDerivatives 4.0

International (CC BY-NC-ND 4.0) License. 